\section{AIAA Journal}

1) A. Kelkar, W. Elber \& I.S. Raju: Large deflection behavior of circular quasi-isotropic laminates under point loading, 25, 1 (1987), 99. *graphite/ epoxy, large deformation, low-velocity impact, plate, membrane coupling.

2) T. Ishikawa, M. Matsushima \& Y. Hayashi: Geometrical and material nonlinear properties of twodimensional fabric composites, 25, 1 (1987), 107. *carbon/epoxy, longitudinal hardening, plain-weave composite, theory, experiment.

3) M. Stein \& D.C. Jegley: Effects of transverse shearing on cylindrical bending, vibration, and buckling of laminated plates, 25, 1 (1987), 123. *thick plate, laminated plate, variational theorem, comparison with experiment.

4) S.V. Rajagopal, G. Singh \& Y.V.K.S. Rao: Large-deflection and nonlinear vibration of multilayered sandwich plates, 25, 1 (1987), 130. *rectangular finite element, all nonlinear terms, number of layers, numerical analysis.

5) R.L. Ramkumar, P.G. Chen \& W.J. Sanders: Free vibration solution for clamped orthotropic plates using Lagrangian multiplier technique, 25, 1 (1987), 146. *orthotropic laminated plate, natural frequency, mode shape, low-velocity impact, comparison with experiment.

6) P.C. Chen \& R.L. Ramkumar: Static and dynamic analysis of clamped orthotropic plates using Lagrangian multiplier technique, 25, 2 (1987), 316. *low velocity impact, hard object, shear deformation, natural frequency, damage.

7) S. Ghang: Effective moduli of composite materials in dynamic problems, 25, 3 (1987), 464. *natural frequency, shear vibration, effective modulus theory, vibration mode.

\section{Composites Science and Technology}

1) A. Spencer: Young's modulus and shear modulus of a composite shaft from resonance measurements, 28, 3 (1987), 173. *axial vibration resonance, torsional resonance, shaft, frequency, comparison with experiment, quality control.

2) J.N. Price \& D. Hull: Effect of matrix toughness on crack propagation during stress corrosion of glass reinforced composites, 28, 3 (1987), 193. *aligned glass fiber, polyester matrix, stress corrosion crack, $\mathrm{HCl}$, fracture mechanics, fractography.

3) J.N. Price \& D. Hull: Axial crushing of glass fibrepolyester composite cones, 28, 3 (1987), 211. * energy absorbing application, truncated conical shell, chopped strand mat, polyester, crush zone morphology, failure micromechanisms.

4) J.G. Ren: Bending of simply-supported, antisymmetrically laminated rectangular plate under transverse loading, 28, 3 (1987), 231. *angle-ply laminate, antisymmetric plate, double Fourier series expansion, analysis, comparison with classical lamination theory.

5) J.A. Barnes, M. Kumosa \& D. Hull: Theoretical and experimental evaluation of the Iosipescu shear test, 28, 4 (1987), 251. *shear property, unidirectional laminate, interlaminar shear strength, shear stiffness, stress distribution, theory, experiment.

6) W.-X. Fan: On phenomenological anisotropic failure criteria, 28, 4 (1987), 269. *refined maximum stress criterion, homogeneous quadratic criterion, strong anisotropy, coupling effect.

7) T.V. Parry, J.M. Wilson \& D.C. Leck: Pull-out steel fasteners from a laminated composite subjected to external pressure, 28, 4 (1987), 279. *single fastener, glass/epoxy composite cylinder, external pressure, friction, analysis, experiment.

8) S. Fischer \& G. Marom: The flexural behaviour of aramid fibre hybrid composite materials, 28, 4 (1987), 291. *elastic-plastic model, shift of neutral axis, aramid/glass, aramid/carbon, sandwich.

9) A.J. Smiley \& R.B. Pipes: Rate sensitivity of mode II interlaminar fracture toughness in graphite/epoxy and graphite/PEEK composite materials, 29, 1 (1987), 1. *end notched flexure, various crosshead speed, fracture mechanism, laminates.

10) A.N. Kumar, R.K. Pandey \& A. Nanda: Crack growth characterization in a short fibre glass-epoxy composite, 29, 1 (1987), 17. *fracture resistance, random short fiber composite, three point bending, tension, effect of dimensional parameters.

11) H. Jiang, C. Wu, A. Zhang \& P. Yang: Structural characteristics of polyacrylonitrile (PAN) fibers during oxidative stabilization, 29, 1 (1987), 33. *structure change, X-ray scanning, various measuring equipment, two-phase structure, PAN fiber.

12) P.A. Smith \& K.J. Pascoe: Fatigue of bolted joints in (0/90) CFRP laminates, 29, 1 (1987), 45. *double-lap bolted joint, fatigue, damage development, stiffness-based failure criterion, bolt clamp-up.

\section{Experimental Mechanics}

1) G. Abu-Farsakh: Experimental buckling of GRP cylindrical shells, 23, 1 (1987), 1. *axial compression, external pressure, errors, geometrical imperfection, measurement procedure. 
2) I.G. Zewi, I.M. Daniel \& J.'T. Gorto: Residual stresses and warpage in woven-glass/epoxy laminates, 23, 1 (1987), 44 . *curing process, thermal expansion, asymmetries, mechanical property, room temperature, high temperature, moire technique.

3) S.E. Groves, C.E. Harris, A.L. Highsmith, D.H. Allen \& R.G. Norvell: An experimental and analytical treatment of matrix cracking in cross-ply laminates, 23, 1 (1987), 73. *damage, crack mechanism, crack growth, stiffness, straight crack, curved crack, stress state, finite element method.

\section{Forest Products Journal}

1) G.A. Eckelman \& S. Munz: Rational design of cases with front frames and semi-rigid joints, 37, 6 (1987), 25. *hinged joint, rigid joint, stiffness, modulus of rigidity, vertical force.

\section{Holz als Roh- und Werkstoff}

1) B. Heimeshoff: Zur Berechnung von Biegeträgern aus nachgiebig miteinander verbundenen Querschnittsteilen im Ingenieurholzbau, 45, 6 (1987), 237. *sinusförmiger Belastung, zusammengesetzen Biegeträger.

2) R.O. Foschi: A procedure for the determination of localized modulus of elasticity, 45, 6 (1987), 257. *compliance, Fourier transform, grading machine, cut-off frequency, average modulus of elasticity.

\section{International Journal of Mechanical Sciences}

1) R. Kitching \& P. Myler: Glass reinforced plastic pipe bend subjected to vacuum and flexure, 28, 3 (1986), 179. *right-angled pipe, chopped strand mat, vacuum, bend, experiment, analysis.

2) S. Adali \& H. Nissen: Micromechanical initial failure analysis of symmetrically laminated cross-ply laminates, 29, 1 (1987), 83. * *first-ply failure, flexural load, void, hygrothermal condition, micromechanics.

\section{International Polymer Science and Technology}

1) V.N. Oborin, S.F. Stus', N.G. Bekin \& F.F. Neplokhov: Formalising the limits for the quality of reinforcement of moulded tyres, 13, 10 (1986), 6 . *moulded tyre, injection-moulding, high viscosity, carcass-cord, control parameter.

2) E.A. Dzyura, L.M. Volchenok, I.V. Markova \& V.P. Zhilko: Methods of varying the stiffness properties of rubber-fibre composites, 13, 10 (1986), 72. *short fiber, disperse filler, polyamide, stiffness properties.

3) O.G. Polyakov, A.I. Kamenshchikov, A.M. Ghaikun \& V.F. Drozdovskii: Effect on the content of short fibres on the strength and change in bulk volume of rubber-fibre composites during stretching, 13, 11 (1986), 21. *short fibre, fibre content, bulk volume, fibre orientation, stretching.

4) A.V. Savel'ev, V.G. Vnukova, V. Ya.Kiselev \& I.A. Tutorskii: Determining the adhesion strength between a filler and elastomer, 13, 11 (1986), 26. *adhesion strength, filler, elastomer, adsorption interaction, metal oxide.

5) P. Hirschberg, G. Novotny, G. Vamos \& D. Kallo: Increasing polyester resin volume; the materialeconomising concept in processing of glass fibrereinforced polyesters, 13, 12 (1986), 21. *glass fibre, polyester resin, integrated structure, tensile strength, flexural modulus.

6) F.I. Duntov, V.D. Rumyantsev, A.N. Karasev, A.L. Gol'denberg, V.M. Belyaev, L.V. Mashkova, G.P. Gradoboeva, T.A. Fadeeva, E.A. Vasilenko, M.N. Shustitskaya \& L.K. Sotnikova: Adhesion strength of copolymers of ethylene and vinyl acetate, 13, 12 (1986), 52. *adhesive layer, metal molecular mass characteristic, temperature, pressure.

7) B.G. Gavrilov, A.M. Uminov, G.L. Portnyi \& I.M. Muslaev: Determining the adhesion of unvulcanised rubber to metal, 13, 12 (1986), 60. *tyre product, metal drum, tyre assembly, radial tyre, tubeless.

\section{Journal of Adhesion}

1) S. Mall: Fructure evaluation of a thermoplastic polyimide-sulfonc as adhesive, 20, 4 (1987), 251. *compliance, fracture energy, molecular weight, lap shear strength, debond length.

\section{Journal of Engineering Mechanics}

1) V.S. Gopalaratnam \& S.P. Shah: Tensile failure of steel fiber-reinforced mortar, 113, 5 (1987), 635. * $1.5 \%$ fibers, SFRC, fiber-matrix interface, tensile test, crack width, single fiber pull-out.

\section{Journal of Structural Engineering}

1) R.J. Craig \& J. Decker: Inelastic behavior of reinforced fibrous concrete, 113, 4 (1987), 802. *steel fibers, moment-deflection curves, strain hardening, tensile stresses.

\section{NASA Contractor Report}

1) J.N. Reddy \& G.F. Liu: A higher-order theory for geometrically nonlinear analysis of composite laminates, CR-4056 (1987). *third-order shear deformation theory, angle-ply, cross-ply, finite element model, vibration, plates and shells.

\section{(The) Quarterly Journal of Mechanics and Applied} Mathematics

1) A.J.M. Spencer: The reinforcement of a hole in a flat plate by cylindrically wound fibre-reinforced disks, 40, 1 (1987), 105. *hoop stress, all-round tension, uniform disks, tapered disks, countersunk disks, pure 
shear.

\section{Tire Science and Technology}

1) K. Yamagishi, M. Togashi, S. Furuya, K. Tsukahara \& N. Yoshimura: A study on the contour of the radial tire: Rolling contour optimization theoryRCOT, 15, 1 (1987), 3. *tire contour, tension distribution, inflation pressure, rolling resistance, riding comfort.

2) E.G. Markow: Evolving the banded radial tire, 15, 1 (1987), 30. *banded tire, rolling resistance, tread wear, puncture, run-flat.

3) R.A. Stechschulte \& J.R. Luchini: A laminated composite solid element and its application to tire analysis, 15, 1 (1987), 42. *finite element, laminated composite, solid element, tire model.

4) R.L. Keefe: Predictive indoor wheel testing of heavy duty truck tires, 15, 1 (1987), 58. *tires, truck tires, tire fatigue, tire tests, indoor tire tests.

紙面の都合上, キーワードをもって概要に代えた. *はキーワードを示す.

（情報委員会）

\section{日本複合材料学会誌（邦文誌）への論文投稿のすすめ}

日本複合材料学会誌 (邦文誌) は年 6 回の刊行で, 各号に $3 \sim 4$ 編の邦文論文（研究論文, 研究ノート, 技術 資料）の掲載を予定しております. 会員各位の積極的なご投稿をおすすめいたします.

投稿資格 本会会員に限りますが，会員外の共同 研究者を含むことはさしつかえありません.

\section{到原稿の種類および制限頁数}

研 究 論 文 : 刷上り 6 ページ以内

研究ノート：刷上り 2 ページ以内

技術資料：刷上り 6 ページ以内

副投稿の申込み任意の用紙に著者名, 論文題目, 論文種目, 論文要旨（400 字以内）および連絡先 等を記入のうえ下記宛郵送ください.

干113 東京都文京区湯島 2-30-9

学会誌刊行センター内

「日本複合材料学会誌」編集委員会

(TEL. 03-817-5825)
折返し，学会所定の原稿用紙（有料）をお送りい たします. 論文作成の要領は原稿用紙の表紙裏面に 印刷してありますのでご参照くたさい。

\section{揭揭載料無料 \\ 別刷料金}

\begin{tabular}{ccccc}
\hline & 50 & 100 & 150 & 200 部 \\
\hline $1 \sim 2$ 頁 & 12,000 & 16,000 & 20,000 & 24,000 円 \\
$3 \sim 4$ & 18,000 & 22,000 & 26,000 & 30,000 \\
$5 \sim 6$ & 24,000 & 28,000 & 32,000 & 36,000 \\
7 & 40,000 & 45,000 & 50,000 & 55,000 \\
8 & 50,000 & 55,000 & 60,000 & 65,000 \\
\hline \multicolumn{4}{r}{} & (全表紙付, 購入義務最少 50 部)
\end{tabular}

